\title{
Rotational stability of tidally deformed planetary bodies
}

\author{
I. Matsuyama ${ }^{1}$ and F. Nimmo ${ }^{2}$
}

Received 11 May 2007; revised 14 August 2007; accepted 7 September 2007; published 7 November 2007.

[1] We consider the true polar wander (rotational variations driven by mass redistribution) of tidally deformed planetary bodies. The rotation pole of bodies without tidal deformation is stabilized by the component of the rotational bulge which retains a memory for prior rotational states, that is, a remnant rotational bulge. For planetary bodies with tidal deformation, the additional stabilizing effect of a remnant tidal bulge results in less permissive excursions of the rotation pole. The magnitude of the load driving reorientation is parameterized by $Q$, the ratio between the degree- 2 gravitational potential of the load and the remnant rotational bulge. Reorientation is favored if the initial load longitude is close to $90^{\circ}$, that is, close to the center of the leading or trailing hemisphere. As an illustration of the new theory, we consider reorientation driven by internal loading on Saturn's moon Enceladus. Small loads $(|Q| \approx 1)$ are inconsistent with significant reorientation because of the small present-day angular separation between the load and the rotation axis. Larger loads $(|Q| \approx 2)$ permit reorientations approaching $90^{\circ}$. Large reorientation scenarios are consistent with the present-day equatorial location of a geologically inferred ancient polar terrain.

Citation: Matsuyama, I., and F. Nimmo (2007), Rotational stability of tidally deformed planetary bodies, J. Geophys. Res., 112, E11003, doi:10.1029/2007JE002942.

\section{Introduction}

[2] External torques acting on the rotational bulge of a planetary body may cause motions of the rotation axis, such as the lunisolar precession of the Earth's rotation axis. Evans [1866] noted that even in the absence of external torques, reorientation of the rotation axis may occur, as viewed by an observer on the surface of the body, due to mass redistribution. For a rigid spinning body, the lowest kinetic energy state for a given angular momentum corresponds to rotation around the principal axis of inertia with the largest moment, hereafter referred to as the maximum principal axis. Hence it is generally assumed that internal energy dissipation will ultimately drive planetary bodies to this state. Mass redistribution leads to reorientation to a new principal axis rotational state, while the angular momentum vector remains fixed in space. This type of reorientation of the rotation axis is commonly referred to as true polar wander (TPW) to distinguish it from apparent polar wander (APW), which refers to the reorientation of the rotation axis relative to the surface due to plate tectonics. On Earth, observations of star positions taken over the last century indicate TPW at a rate of $\sim 1^{\circ} / \mathrm{Myr}$ [Argus and Gross, 2004]. The time averaged magnetic pole position can be

\footnotetext{
${ }^{1}$ Department of Terrestrial Magnetism, Carnegie Institution of Washington, Washington, D. C., USA.

${ }^{2}$ Department of Earth and Planetary Sciences, University of California, Santa Cruz, Santa Cruz, California, USA.

Copyright 2007 by the American Geophysical Union. 0148-0227/07/2007JE002942\$09.00
}

used to infer the secular TPW history. These studies suggest $\sim 30^{\circ}$ of TPW over the last $200 \mathrm{Myr}$ [Besse and Courtillot, 2002], and a $\sim 90^{\circ} \mathrm{TPW}$ event during the Cambrian period [Kirschvink et al., 1997].

[3] We will focus on rotational variations due to TPW. Hereafter, we will describe these variations in a reference frame fixed to the rotating body; hence we will refer to the reorientation of the rotation axis, rather than the reorientation of the body. Evans [1866] described how a mass deficit would "pull" the rotation axis, while a mass excess would "push" the rotation axis using dynamical arguments. Motivated by this study, Darwin [1877] found mathematical TPW solutions by solving the equations for angular momentum conservation, commonly referred to as Euler's equations. Gold [1955] argued that while the rotational bulge stabilizes the rotation axis temporarily, its orientation is ultimately determined by those contributions to the inertia tensor which are not associated with rotation since the rotational bulge eventually adjusts to any reorientation of the rotation axis. Goldreich and Toomre [1969] demonstrated that if mass redistribution occurs slowly (over time scales that are longer than the slowest of the nutations experienced), the solid angle between the rotation axis and the maximum principal axis is an adiabatic invariant. It then follows that once the planet achieves maximum principal axis rotation, it will continue in this configuration and thus mass redistribution drives TPW as the principal axis of inertia migrates. The final rotation axis orientation (after realignment of the hydrostatic figure) can be found by diagonalizing the non-hydrostatic inertia tensor. This approach has been used to study the long-term rotational 
stability of planets [e.g., Melosh, 1980; Steinberger and O'Connell, 1997; Zuber and Smith, 1997; Bills and James, 1999; Sprenke et al., 2005]. Alternatively, given the timedependent mass redistribution, it is possible to find TPW solutions by explicitly solving the angular momentum conservation equation [e.g., Spada et al., 1992, 1996; Ricard et al., 1993; Richards et al., 1997, 1999; Steinberger and O'Connell, 1997; Greff-Lefftz, 2004].

[4] Evans [1866] had noted that the presence of an elastic lithosphere may stabilize the rotation axis since it establishes a memory of prior rotation pole locations, that is, a remnant rotational bulge. Willemann [1984] revisited this issue and found TPW solutions by diagonalizing the inertia tensor associated with the TPW-driving load and the remnant rotational bulge. We will extend the analysis of Willemann [1984] by quantifying the long-term TPW of a satellite with remnant rotational and tidal bulges. The paper is organized as follows. Section 2 focuses on inertia tensor perturbations associated with the effect of mass loads and the deformation driven by changes in the centrifugal and tidal potentials. Section 3 outlines TPW solutions obtained by diagonalizing the inertia tensor. Sections 4 and 5 consider the implications of the new results for estimates of the reorientation of Saturn's moon Enceladus. Finally, section 6 summarizes the main results and discusses some of their consequences.

\section{Inertia Tensor Perturbations}

[5] We assume that the unperturbed, non-rotating planet is spherically symmetric with density $\rho_{0}(r)$. The inertia tensor of the unperturbed planet is given by

$$
I_{0} \delta_{i j}=\frac{8 \pi}{3} \delta_{i j} \int_{0}^{a} d r r^{4} \rho_{0},
$$

where $\delta_{i j}$ is the Kronecker delta and $a$ is the mean radius. We expand the density perturbations, $\delta \rho$, responsible for changes in the inertia tensor in terms of spherical harmonic basis functions, $Y_{\ell m}$, as

$$
\delta \rho(r, \theta, \phi)=\sum_{\ell=0}^{\infty} \sum_{m=-\ell}^{\ell} \rho_{\ell m}(r) Y_{\ell m}(\theta, \phi),
$$

where $\rho_{\ell m}$ are the spherical harmonic coefficients, $\theta$ denotes colatitude, and $\phi$ denotes longitude measured eastward from the $\mathrm{x}$ axis. We adopt the normalization

$$
\int_{S} d S Y_{\ell^{\prime} m^{\prime}}^{\dagger}(\theta, \phi) Y_{\ell m}(\theta, \phi)=4 \pi \delta_{\ell \ell^{\prime}} \delta_{m m^{\prime}}
$$

where $S$ denotes the complete solid angle and $\dagger$ represents the complex conjugate. The inertia tensor perturbations are given by

$$
\delta I_{i j}=\int_{V} d V \delta \rho\left(r^{2} \delta_{i j}-r_{i} r_{j}\right)
$$

where $V$ is the total volume of the planet.

[6] We will consider the special case of density perturbations with azimuthal symmetry. We denote the spherical harmonic coefficients for the density perturbation, if its symmetry axis is at the north pole, as $\rho_{\ell 0}{ }^{\prime}$. We can use the addition theorem for spherical harmonics to write the coefficients for the case where the symmetry axis is at an arbitrary location with spherical coordinates $(\theta, \phi)$ as

$$
\rho_{\ell m}=\frac{\rho_{\ell 0}^{\prime}}{\sqrt{2 \ell+1}} Y_{\ell m}^{\dagger}(\theta, \phi)
$$

Using equations (2)-(5) yields, after some algebra, the following expressions for the associated inertia tensor perturbations:

$$
\delta I_{i j}=I_{00} \delta_{i j}+\left(\frac{1}{3} \delta_{i j}-e_{i} e_{j}\right) \frac{4 \pi}{\sqrt{5}} \int_{0}^{a} d r r^{4} \rho_{20}^{\prime}(r),
$$

where

$$
I_{00}=\frac{8 \pi}{3} \int_{0}^{a} d r r^{4} \rho_{00}
$$

and $e_{i}=(\sin \theta \cos \phi, \sin \theta \sin \phi, \cos \theta)$ is a unit vector aligned with the symmetry axis of the density perturbations.

[7] It is useful to rewrite equation (6) as a function of the gravitational potential associated with the density perturbation. The gravitational potential external to the planet due to a density perturbation, $\delta \rho$, can be expanded in spherical harmonic as

$$
\delta \mathcal{G}(r, \theta, \phi)=\frac{G M}{r} \sum_{\ell=0}^{\infty} \sum_{m=-\ell}^{\ell}\left(\frac{a}{r}\right)^{\ell} \mathcal{G}_{\ell m}(r) Y_{\ell m}(\theta, \phi),
$$

where $G$ is the gravitational constant, $\mathcal{G}_{\ell m}$ are dimensionless spherical harmonic coefficients, and $M$ is the total mass of the planet. The potential coefficients, $\mathcal{G}_{\ell m}$, are related to the density coefficients, $\rho_{\ell m}$, by

$$
\mathcal{G}_{\ell m}=\frac{1}{(2 \ell+1)} \frac{4 \pi}{M a^{\ell}} \int_{0}^{a} d r r^{2+\ell} \rho_{\ell m}(r)
$$

with suitable normalization [Kaula, 1968, p. 67]. Note that $\mathcal{G}_{00}$ drops out if the total mass of the planet is conserved. Replacing equation (8) with $\ell=2$ and $m=0$ in equation (6) yields

$$
\delta I_{i j}=I_{00} \delta_{i j}+\sqrt{5} M a^{2} \mathcal{G}_{20}^{\prime}\left(\frac{1}{3} \delta_{i j}-e_{i} e_{j}\right),
$$

where $\mathcal{G}_{20}^{\prime}$ is the degree- 2 and order-0 potential coefficient for the symmetry axis at the north pole. Equation (9) allows us to obtain expressions for the inertia tensor perturbations associated with any axisymmetric potential perturbation.

[8] We have shown that the inertia tensor of an axisymmetric mass load is uniquely defined by the degree- 0 , and the degree- 2 and order- 0 coefficients due to the orthogonality of the spherical harmonics. It can be shown, using the same orthogonality conditions, that the inertia tensor of any complicated geophysical mass anomaly is uniquely defined by the degree- 0 and degree- 2 harmonic coefficients. 


\subsection{Load Contributions}

[9] As we discussed in the introduction, mass redistribution is the driving force for TPW. We denote the dimensionless gravitational potential coefficient associated with an axisymmetric load, if centered on the north pole, as $\mathcal{G}_{\ell m}^{L \prime}$. Using equation (9), the inertia tensor perturbations associated with this load, if its symmetry axis is at an arbitrary position with spherical coordinates $\left(\theta_{L}, \phi_{L}\right)$, can be written as

$$
\delta I_{i j}^{L}=I_{00}^{L}+\sqrt{5} M a^{2} \mathcal{G}_{20}^{L \prime}\left(\frac{1}{3} \delta_{i j}-e_{i}^{L} e_{j}^{L}\right),
$$

where $I_{00}^{L}$ is a spherically symmetric perturbation associated with a purely radial perturbation, and $e_{i}^{L}=\left(\sin \theta_{L} \cos \phi_{L}\right.$, $\left.\sin \theta_{L} \sin \phi_{L}, \cos \theta_{L}\right)$ is a unit vector aligned with the symmetry axis of the load.

\subsection{Rotational Deformation}

[10] We denote the spherical harmonic coefficients for the centrifugal potential, if the rotation pole is at the north pole, as $\mathcal{G}_{\ell m}^{R \prime}$. Using our normalization for the spherical harmonics, these spherical harmonic coefficients are

$$
\mathcal{G}_{00}^{R \prime}=\frac{a^{3} \Omega^{2}}{3 G M}
$$

and

$$
\mathcal{G}_{20}^{R \prime}=-\frac{a^{3} \Omega^{2}}{3 \sqrt{5} G M},
$$

where $\Omega$ is the angular velocity of the planet. The potential coefficients associated with the long-term response driven by the centrifugal potential can be written as

$$
\mathcal{G}_{\ell m}^{R D \prime}=k_{\ell}^{T} \mathcal{G}_{\ell m}^{R \prime},
$$

where $k_{\ell}^{T}$ is the degree- $\ell$ tidal Love number [Love, 1911]. This dimensionless number describes the response to tidal forcings and depends on the body's interior structure and rheology. Since we are interested in the long-term inertia tensor perturbations, we use the secular (or fluid limit) Love number. That is, we implicitly assume that all viscous stresses have relaxed by using the secular Love number. Using equations (9), (12), and (13), the inertia tensor perturbation associated with the rotational deformation for a rotation pole at an arbitrary location with spherical coordinates $\left(\theta_{R}, \phi_{R}\right)$, is given by

$$
\delta I_{i j}^{R D}=I_{00}^{R D} \delta_{i j}+k_{2}^{T} \frac{a^{5} \Omega^{2}}{3 G}\left(e_{i}^{\Omega} e_{j}^{\Omega}-\frac{1}{3} \delta_{i j}\right) .
$$

In the last equation, $I_{00}^{R D}$ is a spherically symmetric perturbation associated with the planetary response to the radially directed component of the centrifugal force, and $e_{i}^{\Omega}$ $=\left(\sin \theta_{R} \cos \phi_{R}, \sin \theta_{R} \sin \phi_{R}, \cos \theta_{R}\right)$ is a unit vector aligned with the rotation pole.

[11] The initial rotational figure is established early, when there is no lithosphere; hence we assume that the initial figure is hydrostatic [Willemann, 1984]. This is a valid assumption for most planetary bodies; however, it may not be valid for tidally deformed satellites if significant tidal evolution of the orbit (which causes rotation rate variations) occurs. As the planet cools and the lithosphere forms, it may preserve a record of its initial (before reorientation) rotation pole, with spherical coordinates $\left(\theta_{R}^{*}, \phi_{R}{ }^{*}\right)$, in the form of a remnant rotational bulge. The rotation pole is stabilized due to the presence of this remnant rotational bulge since the lithosphere is strained from its equilibrium figure when the rotation pole moves. The inertia tensor perturbation associated with rotational deformation can be written as

$$
\begin{aligned}
\delta I_{i j}^{R}= & I_{00}^{R D} \delta_{i j}+k_{2}^{T *} \frac{a^{5} \Omega^{2}}{3 G}\left(e_{i}^{\Omega *} e_{j}^{\Omega_{*}}-\frac{1}{3} \delta_{i j}\right) \\
& +k_{2}^{T} \frac{a^{5} \Omega^{2}}{3 G}\left[\left(e_{i}^{\Omega} e_{j}^{\Omega}-\frac{1}{3} \delta_{i j}\right)-\left(e_{i}^{\Omega_{*}} e_{j}^{\Omega_{*}}-\frac{1}{3} \delta_{i j}\right)\right],
\end{aligned}
$$

where we use equation (14), $k_{2}^{T *}$ and $k_{2}^{T}$ are the degree-2 secular tidal Love numbers for the planet without and with a lithosphere, respectively, and $e_{i}^{\Omega *}=\left(\sin \theta_{R}{ }^{*} \cos \phi_{R}{ }^{*}, \sin \theta_{R}{ }^{*}\right.$ $\left.\sin \phi_{R}{ }^{*}, \cos \theta_{R}{ }^{*}\right)$ and $e_{i}^{\Omega}$ are unit vectors aligned with the initial and final rotation poles, respectively. We ignore angular velocity variations and we assume that there is no reorientation during the formation of the lithosphere in equation (15). The second term on the right hand side of equation (15) is the perturbation associated with the initial hydrostatic figure, while the third term is the perturbation associated with the planetary response to the change in the centrifugal potential. It will be useful to rewrite equation (15) as

$$
\begin{aligned}
\delta I_{i j}^{R}= & I_{00}^{R D} \delta_{i j}+\frac{a^{5} \Omega^{2}}{3 G}\left(k_{2}^{T *}-k_{2}^{T}\right)\left(e_{i} * e_{j}{ }^{2 *}-\frac{1}{3} \delta_{i j}\right) \\
& +\frac{a^{5} \Omega^{2}}{3 G} k_{2}^{T}\left(e_{i}^{\Omega} e_{j}^{\Omega}-\frac{1}{3} \delta_{i j}\right),
\end{aligned}
$$

where we can identify the second term on the right hand side of equation (16) as the remnant rotational bulge. Consider the case of a planet in which a lithosphere does not form. In this case, $k_{2}^{T *}=k_{2}^{T}$ and equations (15) and (16) simplify to equation (14), as would be expected.

\subsection{Tidal Deformation}

[12] We denote the spherical harmonic coefficients for the tidal potential, if the tidal axis is aligned with the $\mathrm{z}$ axis of our reference frame, as $\mathcal{G}_{\ell m}^{T \prime}$. The potential coefficients associated with the long-term tidal deformational of the planet are given by

$$
\mathcal{G}_{\ell m}^{T D \prime}=k_{\ell}^{T} \mathcal{G}_{\ell m}^{T \prime}
$$

Since we are interested in the long-term inertia tensor perturbation, we assume that the planet has been driven to synchronous rotation. In this case the tidal potential has the same form as the centrifugal potential, but with a different axis of symmetry and magnitude [Murray and Dermott, 1999, section 4.7]:

$$
\mathcal{G}_{20}^{T \prime}=-3 \mathcal{G}_{20}^{R \prime}=\frac{a^{3} \Omega^{2}}{\sqrt{5} G M} .
$$


Using equations (9), (17), and (18), the inertia tensor perturbation associated with tidal deformation for a tidal axis at an arbitrary location with spherical coordinates $\left(\theta_{T}\right.$, $\left.\phi_{T}\right)$ is given by

$$
\delta I_{i j}^{T D}=I_{00}^{T D} \delta_{i j}-k_{2}^{T} \frac{a^{5} \Omega^{2}}{G}\left(e_{i}^{T} e_{j}^{T}-\frac{1}{3} \delta_{i j}\right) .
$$

In this expression, $I_{00}^{T D}$ is a spherically symmetric perturbation associated with the planetary response to the radially directed component of the tidal force, and $e_{i}^{T}=\left(\sin \theta_{T} \cos \phi_{T}\right.$, $\left.\sin \theta_{T} \sin \phi_{T}, \cos \theta_{T}\right)$ is a unit vector aligned with the tidal axis. Once again, the initial figure of the planet associated with tidal deformation is hydrostatic, and the formation of a lithosphere as the planet cools results in a remnant tidal bulge. The inertia tensor perturbation associated with tidal deformation can be written as

$$
\begin{aligned}
\delta I_{i j}^{T}= & I_{00}^{T D} \delta_{i j}-k_{2}^{T *} \frac{a^{5} \Omega^{2}}{G}\left(e_{i}^{T_{*}} e_{j}^{T_{*}}-\frac{1}{3} \delta_{i j}\right) \\
& -k_{2}^{T} \frac{a^{5} \Omega^{2}}{G}\left[\left(e_{i}^{T} e_{j}^{T}-\frac{1}{3} \delta_{i j}\right)-\left(e_{i}^{T *} e_{j}^{T *}-\frac{1}{3} \delta_{i j}\right)\right],
\end{aligned}
$$

where we use equation (19), and $e_{i}^{T *}$ and $e_{i}^{T}$ are unit vectors aligned with the initial and final tidal axes, respectively. The second term on the right-hand side of equation (20) is the initial hydrostatic perturbation, and the third term is the perturbation associated with the planetary response to the change in the tidal potential. We rewrite equation (20) as

$$
\begin{aligned}
\delta I_{i j}^{T}= & I_{00}^{T D} \delta_{i j}-\frac{a^{5} \Omega^{2}}{G}\left(k_{2}^{T_{*}}-k_{2}^{T}\right)\left(e_{i}^{T_{*}} e_{j}^{T_{*}}-\frac{1}{3} \delta_{i j}\right) \\
& -\frac{a^{5} \Omega^{2}}{G} k_{2}^{T}\left(e_{i}^{T} e_{j}^{T}-\frac{1}{3} \delta_{i j}\right),
\end{aligned}
$$

where we can identify the second term on the right-hand side as the remnant tidal bulge. Once again, if we consider the special case of a hydrostatic planet, then $k_{2}^{T *}=k_{2}^{T}$ and equations (20) and (21) simplify to equation (19), as would be expected.

\subsection{Total Inertia Tensor Perturbations}

[13] We denote the total inertia tensor including perturbations due to an axisymmetric load, and rotational and tidal deformation as $I_{i j}=I_{0} \delta_{i j}+\delta I_{i j}^{L}+\delta I_{i j}^{R}+\delta I_{i j}^{T}$. Using equations (10), (16), and (21) yields

$$
\begin{aligned}
I_{i j}= & I \delta_{i j}+M a^{2}\left\{( m ^ { * } - m ) \left[Q\left(\frac{1}{3} \delta_{i j}-e_{i}^{L} e_{j}^{L}\right)\right.\right. \\
& \left.+\left(e_{i}^{\Omega *} e_{j}^{\Omega *}-\frac{1}{3} \delta_{i j}\right)-3\left(e_{i}^{T *} e_{j}^{T *}-\frac{1}{3} \delta_{i j}\right)\right] \\
& \left.+m\left[\left(e_{i}^{\Omega} e_{j}^{\Omega}-\frac{1}{3} \delta_{i j}\right)-3\left(e_{i}^{T} e_{j}^{T}-\frac{1}{3} \delta_{i j}\right)\right]\right\} ;
\end{aligned}
$$

where

$$
I \equiv I_{0}+I_{00}^{L}+I_{00}^{R D}+I_{00}^{T D}
$$

and we define the dimensionless numbers

$$
\begin{gathered}
m^{*} \equiv k_{2}^{T *} \frac{\Omega^{2} a^{3}}{3 G M}, \\
m \equiv k_{2}^{T} \frac{\Omega^{2} a^{3}}{3 G M},
\end{gathered}
$$

and

$$
Q \equiv \frac{\sqrt{5} \mathcal{G}_{20}^{L \prime}}{m^{*}-m} .
$$

The parameter $Q$, first introduced by Willemann [1984] and given by the ratio of the degree- 2 gravitational potential perturbation of the load and the remnant rotational bulge, is a useful normalization for the load size. This parameter is positive/negative for a mass excess/deficit with our sign convention for the gravitational potential (equation (8)) since $k_{2}^{T *}-k_{2}^{T}$ (and thus $m^{*}-m$ ) is always positive.

\section{TPW Solutions From Diagonalization of the Inertia Tensor}

[14] Tidal torques eventually drive planetary bodies to synchronous rotation, in which the minimum principal axis is aligned with the tidal axis. Hence the lowest kinetic energy state for planetary bodies with tidal deformation corresponds to synchronous rotation, with the minimum and maximum principal axes aligned with the tidal and rotation axes, respectively. We assume that the planet ultimately reaches this minimum energy state, and thus we find the orientation of the rotation and tidal axes by diagonalizing the inertia tensor.

[15] For planets without lithospheres, TPW solutions can be found by diagonalizing the non-hydrostatic inertia tensor since the hydrostatic contribution ultimately adjusts to any new rotation axis orientation [Gold, 1955]. However, the splitting of the inertia tensor into hydrostatic and nonhydrostatic components is not useful for planets with lithospheres since the lithospheric portion of the planet may not behave like a fluid over the time scales associated with the TPW-driving mass redistribution. In this case, the remnant rotational and tidal bulges provide inertia tensor contributions, in addition to the load contributions, which cannot adjust to a new rotation axis orientation. The rotation pole can be found by diagonalizing the component of the inertia tensor perturbation which does not follow the rotation and tidal axes, and we will refer to this contribution as the nonequilibrium inertia tensor.

[16] We use equation (22) to write the non-equilibrium (hence the superscript "NE") inertia tensor as

$$
\begin{aligned}
I_{i j}^{N E}= & M a^{2}\left(m^{*}-m\right)\left[Q\left(\frac{1}{3} \delta_{i j}-e_{i}^{L} e_{j}^{L}\right)\right. \\
& \left.+\left(e_{i}^{\Omega_{*}} e_{j} \Omega_{*}-\frac{1}{3} \delta_{i j}\right)-3\left(e_{i}^{T_{*}} e_{j}^{T *}-\frac{1}{3} \delta_{i j}\right)\right],
\end{aligned}
$$



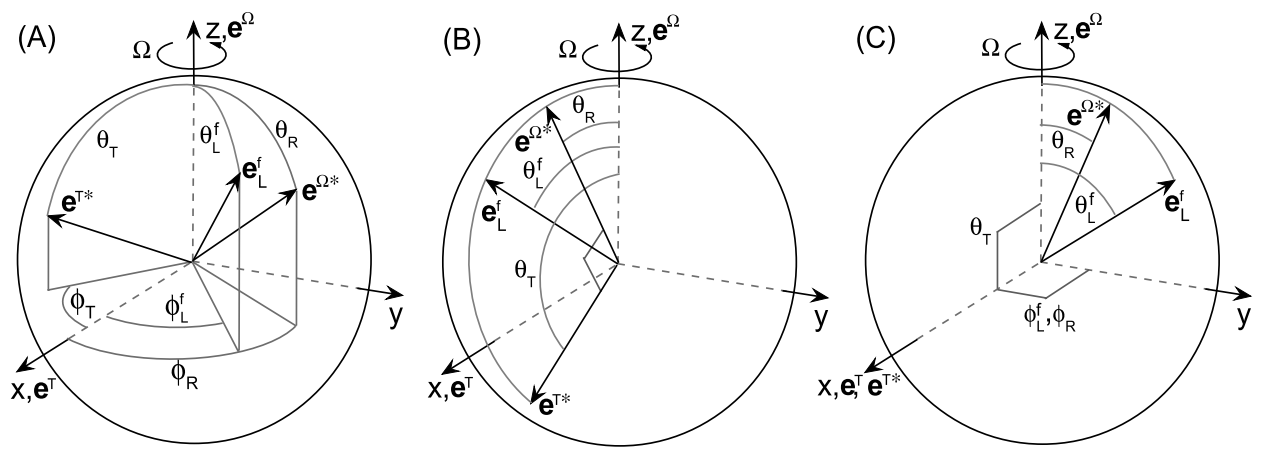

Figure 1. Reorientation in the principal axes reference frame. The final tidal $\left(\mathbf{e}^{T}\right)$ and rotation $\left(\mathbf{e}^{\Omega}\right)$ axes are aligned with the $\mathrm{x}$ and $\mathrm{z}$ axes, respectively. The spherical coordinates for the unit vectors representing the load, $\mathbf{e}_{L}^{f}$, the initial rotation axis, $\mathbf{e}^{\Omega *}$, and the initial tidal axis, $\mathbf{e}^{T *}$, are $\left(\theta_{L}^{f}, \phi_{L}^{f}\right),\left(\theta_{R}, \phi_{R}\right)$, and $\left(\theta_{T}, \phi_{T}\right)$, respectively. (a) Reorientation around an arbitrary axis. (b) Reorientation driven by a positive load around the y axis. In this case, $\phi_{L}^{f}=\phi_{T}=\phi_{R}=0$ and $\theta_{T}=\theta_{R}+90^{\circ}$. (c) Reorientation driven by a positive load around the tidal axis. In this case, $\theta_{T}=\phi_{L}^{f}=\phi_{R}=90^{\circ}$ and $\phi_{T}=0^{\circ}$.

where we ignore the terms associated with the final centrifugal and tidal potentials. The three terms inside the square bracket of equation (27) are associated with the load, the remnant rotational bulge, and the remnant tidal bulge, respectively. We also ignore the spherically symmetric term, $I \delta_{i j}$, in equation (22) since this term will have no effect on the orientation and ordering of the principal axes, and thus it will not impact the reorientation of the planet.

\subsection{Reference Frame Aligned With the Initial Rotation and Tidal Axes}

[17] Let us consider the TPW solutions in a reference frame aligned with the initial rotation and tidal axes. We choose a reference frame where the tidal and rotation axes are directed along the $\mathrm{x}$ and $\mathrm{z}$ axes, respectively. The unit vectors associated with the initial rotation pole, the initial tidal axis, and the symmetry axis of the load are given by $e_{i}^{\Omega *}=(0,0,1), e_{i}^{T *}=(1,0,0)$, and $e_{i}^{L}=\left(\sin \theta_{L} \cos \phi_{L}, \sin \theta_{L} \sin \phi_{L}\right.$, $\left.\sin \phi_{L}, \cos \theta_{L}\right)$, respectively, where $\left(\theta_{L}, \phi_{L}\right)$ are the spherical coordinates for the load. Replacing these unit vectors in the non-equilibrium inertia tensor (equation (27)) yields

$I_{11}^{N E}=M a^{2}\left(m^{*}-m\right)\left[Q\left(1 / 3-\sin ^{2} \theta_{L} \cos ^{2} \phi_{L}\right)-7 / 3\right]$

$I_{22}^{N E}=M a^{2}\left(m^{*}-m\right)\left[Q\left(1 / 3-\sin ^{2} \theta_{L} \sin ^{2} \phi_{L}\right)+2 / 3\right]$

$I_{33}^{N E}=M a^{2}\left(m^{*}-m\right)\left[Q\left(1 / 3-\cos ^{2} \theta_{L}\right)+5 / 3\right]$

$I_{12}^{N E}=-M a^{2}\left(m^{*}-m\right)\left[Q \sin ^{2} \theta_{L} \sin \left(2 \phi_{L}\right) / 2\right]$

$I_{13}^{N E}=-M a^{2}\left(m^{*}-m\right)\left[Q \sin \left(2 \theta_{L}\right) \cos \left(\phi_{L}\right) / 2\right]$

$I_{23}^{N E}=-M a^{2}\left(m^{*}-m\right)\left[Q \sin \left(2 \theta_{L}\right) \sin \left(\phi_{L}\right) / 2\right]$.

[18] For a given load size, $Q$, and initial load location, given by $\theta_{L}$ and $\phi_{L}$, we can find the maximum principal axis orientation by diagonalizing the non-equilibrium inertia tensor using equation (28). The maximum principal axis must coincide with the final rotation axis if the planetary body has achieved the minimum energy state of principal axis rotation. Diagonalizing the inertia tensor yields two possible TPW solutions since choosing the antipole of a given rotation pole solution yields the same solution. The smaller reorientation angle is physically more meaningful since it requires less work.

\subsection{Reference Frame Aligned With the Final Rotation and Tidal Axes}

[19] In many cases the initial load location is unknown but the final location (i.e., the present-day location) is known. Hence we will consider TPW solutions in a reference frame aligned with the final rotation and tidal axes. We assume that the planet has achieved the minimum energy state of principal axis rotation and thus the non-equilibrium inertia tensor must be diagonal. We choose a reference frame where the minimum, intermediate, and maximum principal axes are directed along the $\mathrm{x}, \mathrm{y}$, and $\mathrm{z}$ axes, respectively. We denote the spherical coordinates for the load, the initial rotation axis, and the initial tidal axis as $\left(\theta_{L}^{f}\right.$, $\left.\phi_{L}^{f}\right),\left(\theta_{R}, \phi_{R}\right)$ and $\left(\theta_{T}, \phi_{T}\right)$, respectively, as shown in Figure 1a. The unit vectors representing the load, the initial rotation axis, and the initial tidal axis are given by

$$
\begin{aligned}
e_{i}^{L} & =\left(\sin \theta_{L}^{f} \cos \phi_{L}^{f}, \sin \theta_{L}^{f} \sin \phi_{L}^{f}, \cos \theta_{L}^{f}\right) \\
e_{i}^{\Omega_{*}} & =\left(\sin \theta_{R} \cos \phi_{R}, \sin \theta_{R} \sin \phi_{R}, \cos \theta_{R}\right) \\
e_{i}^{T_{*}} & =\left(\sin \theta_{T} \cos \phi_{T}, \sin \theta_{T} \sin \phi_{T}, \cos \theta_{T}\right) .
\end{aligned}
$$

[20] The initial tidal and rotation axes must be perpendicular to each other. Hence

$$
e_{i}^{T_{*}} \cdot e_{i}^{\Omega_{*}}=\sin \theta_{T} \sin \theta_{R} \cos \left(\phi_{R}-\phi_{T}\right)+\cos \theta_{T} \cos \theta_{R}=0 .
$$

Replacing equations (29)-(31) in equation (27) and setting the off-diagonal components of the non-equilibrium inertia tensor to zero yields

$$
\begin{gathered}
Q \sin ^{2} \theta_{L}^{f} \sin \left(2 \phi_{L}^{f}\right)=\sin ^{2} \theta_{R} \sin \left(2 \phi_{R}\right)-3 \sin ^{2} \theta_{T} \sin \left(2 \phi_{T}\right) \\
Q \sin \left(2 \theta_{L}^{f}\right) \cos \left(\phi_{L}^{f}\right)=\sin \left(2 \theta_{R}\right) \cos \phi_{R}-3 \sin \left(2 \theta_{T}\right) \cos \phi_{T} \\
Q \sin \left(2 \theta_{L}^{f}\right) \sin \left(\phi_{L}^{f}\right)=\sin \left(2 \theta_{R}\right) \sin \phi_{R}-3 \sin \left(2 \theta_{T}\right) \sin \phi_{T}
\end{gathered}
$$


Equations (32)-(35) correspond to four equations for the four unknowns $\theta_{R}, \phi_{R}, \theta_{T}$, and $\phi_{T}$ if we specify the load size, $Q$, and its final location, $\theta_{L}^{f}$ and $\phi_{L}^{f}$. Note that the amount of TPW is given by $\theta_{R}$ (Figure 1a). Once again, diagonalizing the inertia tensor leads to two possible TPW solutions and the solution with the smaller reorientation angle is physically more meaningful since it requires less work.

[21] For a given solution we must verify that $I_{33}^{N E}$ and $I_{11}^{N E}$ correspond to the maximum and minimum principal axes, respectively. Replacing equations (29)-(31) in equation (27), the principal moments relations, $I_{33}^{N E} \geq I_{11}^{N E}, I_{33}^{N E} \geq$ $I_{22}^{N E}$, and $I_{22}^{N E} \geq I_{11}^{N E}$ become

$$
\begin{aligned}
0 \leq & Q\left(\sin ^{2} \theta_{L}^{f} \cos ^{2} \phi_{L}^{f}-\cos ^{2} \theta_{L}^{f}\right)+\cos ^{2} \theta_{R} \\
& -\sin ^{2} \theta_{R} \cos ^{2} \phi_{R}-3 \cos ^{2} \theta_{T}+3 \sin ^{2} \theta_{T} \cos ^{2} \phi_{T}
\end{aligned}
$$

$$
\begin{aligned}
0 \leq & Q\left(\sin ^{2} \theta_{L}^{f} \sin ^{2} \phi_{L}^{f}-\cos ^{2} \theta_{L}^{f}\right)+\cos ^{2} \theta_{R} \\
& -\sin ^{2} \theta_{R} \sin ^{2} \phi_{R}-3 \cos ^{2} \theta_{T}+3 \sin ^{2} \theta_{T} \sin ^{2} \phi_{T}
\end{aligned}
$$

and

$$
\begin{aligned}
0 \leq & Q \sin ^{2} \theta_{L}^{f} \cos \left(2 \phi_{L}^{f}\right)-\sin ^{2} \theta_{R} \cos \left(2 \phi_{R}\right) \\
& +3 \sin ^{2} \theta_{T} \cos \left(2 \phi_{T}\right)
\end{aligned}
$$

respectively.

[22] Using equations (32), (34), and (35) yields, after some algebra,

$$
Q \frac{\cos \left(\phi_{R}-\phi_{L}^{f}\right)}{\left(1+3 \cos ^{2} \theta_{T} / \sin ^{2} \theta_{R}\right)} \sin \left(2 \theta_{L}^{f}\right)=\sin \left(2 \theta_{R}\right)
$$

and

$$
Q \sin \left(\phi_{L}^{f}-\phi_{T}\right) \sin \left(2 \theta_{L}^{f}\right)=\sin \left(\phi_{R}-\phi_{T}\right) \sin \left(2 \theta_{R}\right)
$$

Comparison of equation (39) with TPW solutions for planetary bodies without tidal deformation [e.g., Matsuyama et al., 2006, equation (34)] shows that the effect of tidal deformation may be quantified as a reduction of the TPW driving load size by a factor of $\cos \left(\phi_{R}-\phi_{L}^{f}\right) /\left(1+3 \cos ^{2} \theta_{T} /\right.$ $\left.\sin ^{2} \theta_{R}\right)$. Equation (40) illustrates that moving the load further away from the longitude of the tidal axis (i.e., increasing $\phi_{L}^{f}-\phi_{T}$ ) promotes reorientation.

[23] It is useful to consider some simplifying cases. If reorientation occurs along the meridian passing through the tidal axis (Figure 1b) $\phi_{T}=\phi_{R}=\phi_{L}^{f}=0^{\circ}, \theta_{T}=\theta_{R}+90^{\circ}$, all the terms in equations (33), (35), and (40) vanish, and equations (34) and (39) become

$$
Q \sin \left(2 \theta_{L}^{f}\right)=4 \sin \left(2 \theta_{R}\right)
$$

On the other hand, if reorientation occurs along the meridian perpendicular to the tidal axis (Figure 1c), $\phi_{T}=0^{\circ}, \theta_{T}=\phi_{L}^{f}=$
$\phi_{R}=90^{\circ}$, all the terms in equations (33) and (34) vanish, and equations (35), (39) and (40) become

$$
Q \sin \left(2 \theta_{L}^{f}\right)=\sin \left(2 \theta_{R}\right)
$$

Equations (41) and (42) illustrate that reorientation is more easily accomplished if it occurs around the meridian perpendicular to the tidal axis, as would be expected, since the remnant tidal bulge remains fixed in this case. Note that we have assumed a positive load which drives TPW by pushing the rotation axis away (Figures $1 \mathrm{~b}$ and $1 \mathrm{c}$ ). It can be shown, using similar physical and geometric arguments, that equations (41) and (42) also hold for a negative load which pulls the rotation axis. The TPW solutions for the two specific cases considered above are consistent with the solutions given by equation (1) of Nimmo and Pappalardo [2006].

\section{Results}

[24] Since we considered positive loads in the previous section and we will consider the specific case of TPW on Enceladus driven by an internal mass anomaly, we will focus on negative loads. It is instructive to consider load sizes, $Q$, ranging from -4 to -1 since the lower bound, $|Q|=1$, corresponds to a load with the same size as the remnant rotational bulge, and the upper bound, $|Q|=4$, corresponds to a load with the same size as the combination of the remnant rotational and tidal bulges. We adopt a reference frame aligned with the initial (before reorientation) rotation and tidal axes. Given the load size and the spherical coordinates for its initial location, $\theta_{L}$ and $\phi_{L}$, we can diagonalize the non-equilibrium inertia tensor using equation (28) to find the maximum principal axis, which is assumed to be aligned with the final rotation axis if the planet has achieved the minimum energy state of principal axis rotation.

[25] Figure 2 shows the location of the final rotation axis for a load with $Q=-2$ at different initial locations. The final rotation axis orientation is governed by a balance between the load-induced pull to the rotation axis and stabilization by the remnant tidal and rotational bulges. Stabilization by the remnant rotational bulge is independent of the load longitude since the remnant rotational bulge is axially symmetric around the $\mathrm{z}$ axis and reorientation occurs along meridians. In contrast, stabilization by the remnant tidal bulge depends on the initial load longitude since the remnant tidal bulge is not axially symmetric around the $\mathrm{z}$ axis. Stabilization by the remnant tidal bulge is maximum for $\phi_{L}=0^{\circ}$ (Figures 2a, 2e, and 2i) and zero for $\phi_{L}=90^{\circ}$ (Figures 2d, 2h, and 21). Hence reorientation is more easily accomplished around the tidal axis $\left(\phi_{L}=90^{\circ}\right.$, Figures $2 \mathrm{~d}$, $2 \mathrm{~h}$, and 2l), as would be expected. Unlike the case for reorientation of planetary bodies without tidal deformation [e.g., Willemann, 1984; Matsuyama et al., 2006], reorientation does not generally occur along the great circle connecting the load with the initial rotation axis; rather, it occurs near the meridian perpendicular to the tidal axis due to the dominance of the remnant tidal bulge.

[26] Figure 3 shows the TPW angle and the final angular separation between the load and the rotation axis as a 


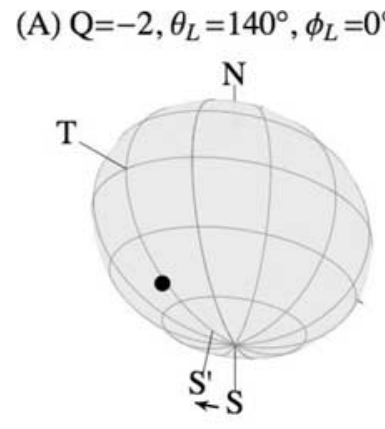

(E) $\mathrm{Q}=-2, \theta_{L}=120^{\circ}, \phi_{L}=0^{\circ}$

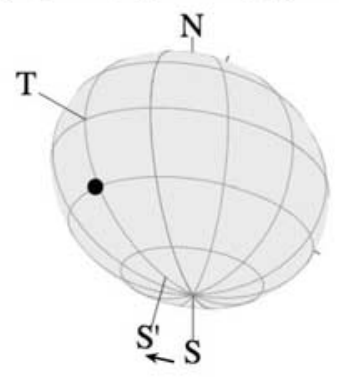

(I) $\mathrm{Q}=-2, \theta_{L}=100^{\circ}, \phi_{L}=0^{\circ}$

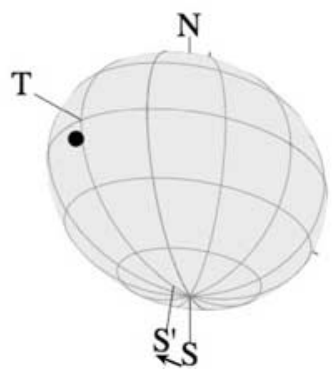

(B) $\mathrm{Q}=-2, \theta_{L}=140^{\circ}, \phi_{L}=30^{\circ}$

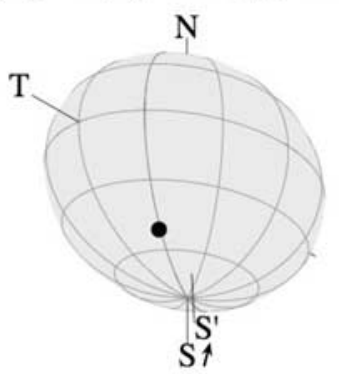

(F) $\mathrm{Q}=-2, \theta_{L}=120^{\circ}, \phi_{L}=30^{\circ}$

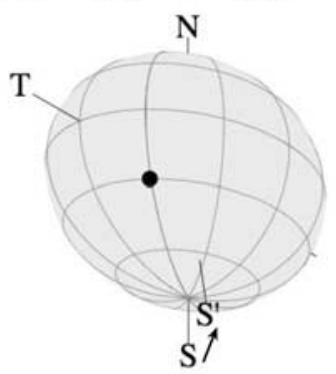

(J) $\mathrm{Q}=-2, \theta_{L}=100^{\circ}, \phi_{L}=30^{\circ}$

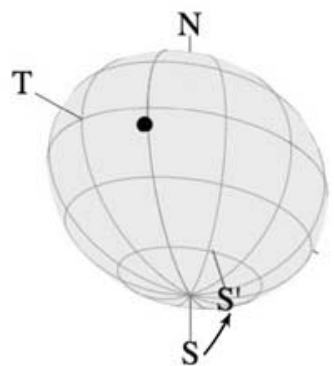

(C) $\mathrm{Q}=-2, \theta_{L}=140^{\circ}, \phi_{L}=60^{\circ}$

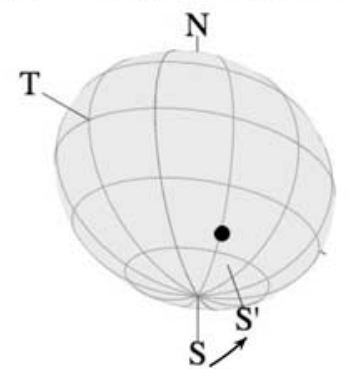

(G) $\mathrm{Q}=-2, \theta_{L}=120^{\circ}, \phi_{L}=60^{\circ}$

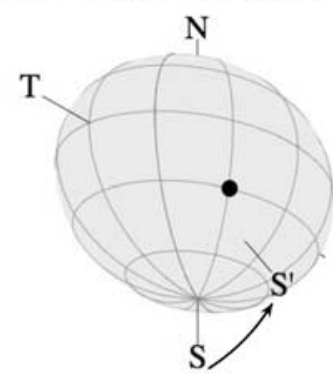

(K) $\mathrm{Q}=-2, \theta_{L}=100^{\circ}, \phi_{L}=60^{\circ}$

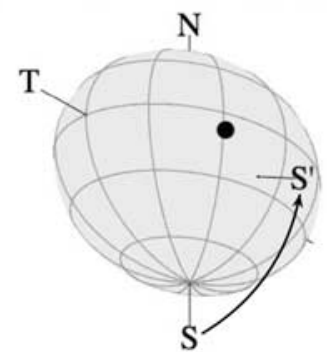

(D) $\mathrm{Q}=-2, \theta_{L}=140^{\circ}, \phi_{L}=90^{\circ}$

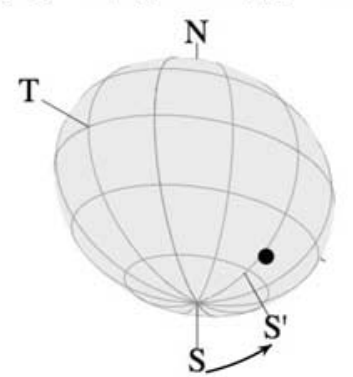

(H) $\mathrm{Q}=-2, \theta_{L}=120^{\circ}, \phi_{L}=90^{\circ}$

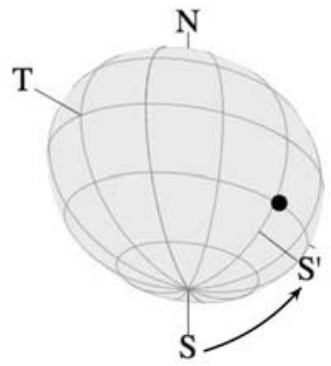

(L) $\mathrm{Q}=-2, \theta_{L}=100^{\circ}, \phi_{L}=90^{\circ}$

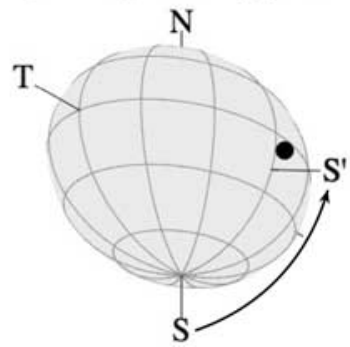

Figure 2. Reorientation of a tidally deformed planetary body driven by an internal load (filled circle) with $Q=-2$ at different initial load locations (given by its spherical coordinates $\theta_{L}$ and $\phi_{L}$, as labeled). Grid lines indicate latitude and longitude in $30^{\circ}$ increments. The reference frame is aligned with the initial (before reorientation) rotation and tidal axes. The initial north pole, south pole, and tidal axis are labeled with the letters $\mathrm{N}, \mathrm{S}$, and T, respectively, and the final (after reorientation) south pole is labeled with the letter $\mathrm{S}^{\prime}$. The arrow indicates the direction of reorientation. Since the load is in the southern hemisphere $\left(\theta_{L}>90^{\circ}\right)$, the angular distance between the load and the rotation axis is given by $180^{\circ}-\theta_{L}$. The load longitude, $\phi_{L}$, is measured eastward from the initial tidal axis, as labeled.

function of the initial angular separation for different load sizes $Q$, and initial load longitudes, $\phi_{L}$. For a given initial angular separation, the smallest TPW angles corresponds to $\phi_{L}=0^{\circ}$ in all cases because both the rotational and tidal remnant bulges fully contribute to the stabilization of the rotation axis in this case. Since $|Q|=4$ corresponds to a load with the same size as the combination of these remnant bulges (the remnant tidal bulge is larger than the remnant rotational bulge by a factor of 3 (equation (18))), the TPW angle is half the initial angular separation for $Q=-4$ and $\phi_{L}=0^{\circ}$ (Figure 3a). Equivalently, the final angular separation between the load and the rotation axis is half the initial angular separation in this case (Figure 3d). As the longitude of the load increases, the stabilizing effect of the tidal remnant bulge diminishes, the TPW angle increases (Figure 3, top panels), and the final angular separation decreases (Figure 3, bottom panels). Once again, reorientation around the tidal axis $\left(\phi_{L}=90^{\circ}\right)$ permits larger excursions of the rotation pole. For a given initial load position, the TPW angle increases, and the final angular separation decreases for negatively larger loads, as would be expected (compare left, middle, and right panels of Figure 3). For $Q=-4$, the final angular distance between the load and the rotation axis is $\leq 7^{\circ}$ if $\phi_{L}=90^{\circ}, \leq 14^{\circ}$ if $\phi_{L} \geq 60^{\circ}$, and $\leq 26^{\circ}$ if $\phi_{L} \geq 30^{\circ}$, for any initial angular distance (Figure $3 \mathrm{~d}$ ). For $Q=-2$, the final angular distance between the load and the rotation axis is $\leq 15^{\circ}$ if $\phi_{L}=90^{\circ}$, and $\leq 25^{\circ}$ if $\phi_{L} \geq 60^{\circ}$, for any initial angular separation (Figure $3 \mathrm{e}$ ).

[27] It is useful to consider the case for $Q=-1$ and reorientation around the tidal axis $\left(\phi_{L}=90^{\circ}\right)$. In this case, the remnant tidal bulge does not contribute to the stabilization of the rotation axis and the final rotation axis orientation is governed by a balance between the loadinduced pull and stabilization by the remnant rotational bulge alone. Since $|Q|=1$ corresponds to a load with the same size as the remnant rotational bulge, the TPW angle 

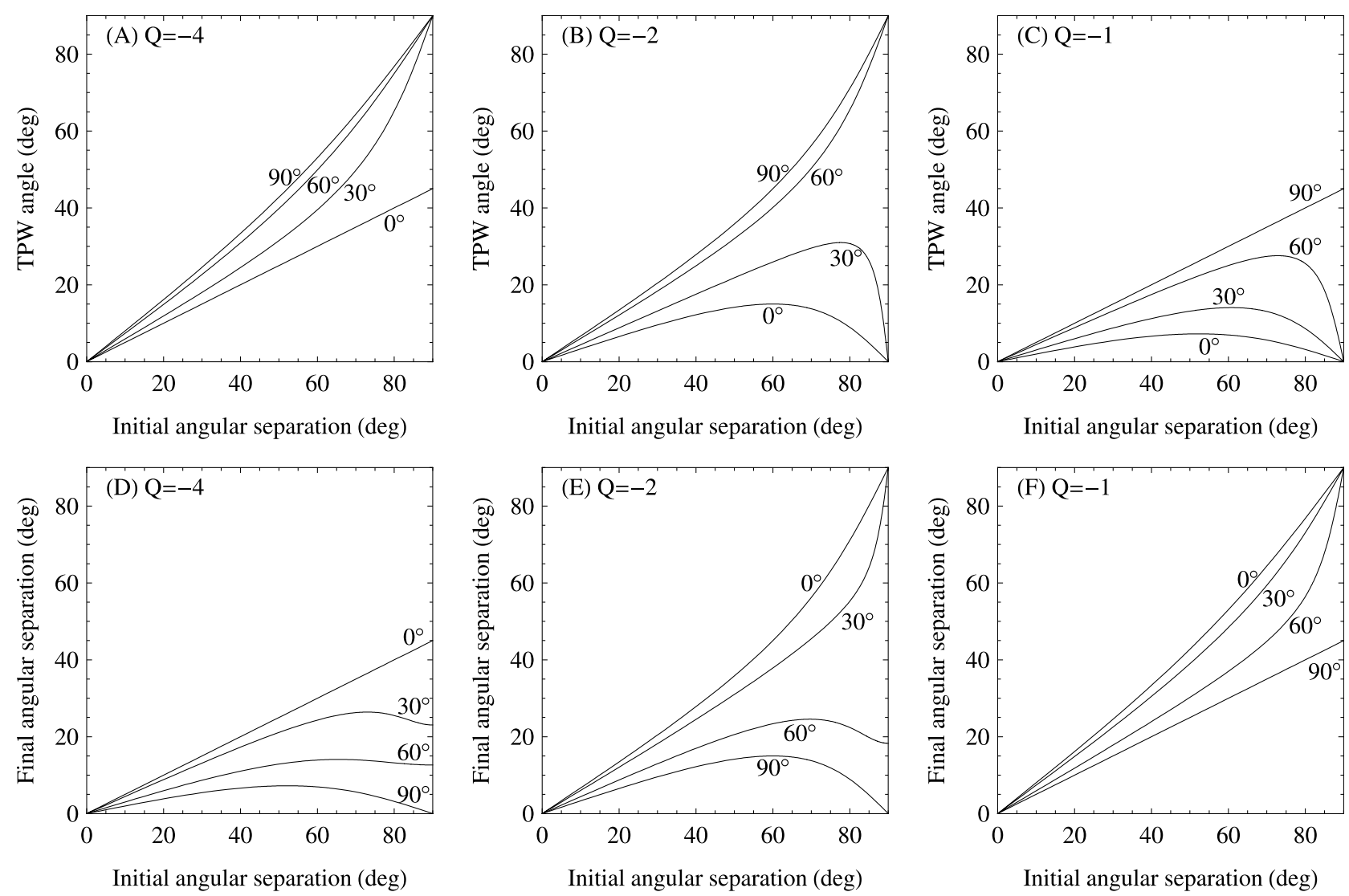

Figure 3. (top) TPW angle and (bottom) final angular separation between the load and the rotation axis as a function of the initial angular separation for different load sizes (left, $Q=-4$; middle, $Q=-2$; right, $Q=-1$ ) and initial load longitudes, $\phi_{L}$, measured eastward from the initial tidal axis (as labeled on each curve).

is half the initial angular separation between the load and the rotation axis (Figure 3c), or equivalently, the final angular separation is half the initial angular separation (Figure 3f). The final angular separation increases as the load longitude decreases from $\phi_{L}=90^{\circ}$ since the remnant tidal bulge provides additional stabilization of the rotation axis in this case. Hence, for $Q=-1$, the final angular separation is at least half the initial separation for any initial load location.

\section{Application to Enceladus}

[28] Having derived the general behavior of satellite reorientation, we will now examine the specific example of Enceladus. The Cassini spacecraft has identified active jets of icy particles and water vapor at the south pole of Enceladus [Porco et al., 2006], an area which is also characterized by recent tectonic deformation and high heat fluxes [Spencer et al., 2006]. The polar location of this hot spot may be explained by reorientation of Enceladus due to TPW [Nimmo and Pappalardo, 2006]. These authors considered the reorientation of Enceladus driven by a lowdensity internal load located at the most favorable location $\left(90^{\circ}\right.$ away from the tidal axis; $n=1$ in their equation (1)). Here we will extend their study by considering reorientation due to a load located at an arbitrary initial position.
[29] There are currently no observational constraints on the size of the load, though these may be determined in future from gravity measurements. Accordingly, we will consider loads with $Q=-1$, corresponding to a load with the same size as the remnant rotational bulge, and $Q=-2$, which is an upper bound based on Figure 2 of Nimmo and Pappalardo [2006]. Another important observational constraint is the present-day angular separation between the load and rotation axis. Inspection of the infra-red thermal anomaly [Spencer et al., 2006, Figure 2] suggests that the center of this thermal anomaly is no more than $8^{\circ}$ distant from the rotation pole. In the absence of other information, we will simply assume that the thermal anomaly location serves as a proxy for the gravity anomaly.

[30] For a load with $Q=-1$ and a final angular separation of $8^{\circ}$, Figure $3 \mathrm{f}$ shows that the initial angular separation can be no more than $16^{\circ}$ and the corresponding TPW less than $8^{\circ}$ (Figure 3c), irrespective of the longitude of the original load. Thus in this case significant TPW cannot have occurred. On the other hand, for a load with $Q=-2$, Figures $3 \mathrm{~b}$ and $3 \mathrm{e}$ show that an initial angular separation of $\geq 81^{\circ}$ is permitted, with a correspondingly large TPW $\left(\geq 73^{\circ}\right)$, for an initial load longitude of $90^{\circ}$. Thus, if the load was sufficiently large, and originally optimally located for reorientation (that is, at the center of the leading or trailing hemispheres), then TPW approaching $90^{\circ}$ can occur. 
(A) Initial rotation and tidal axes reference frame

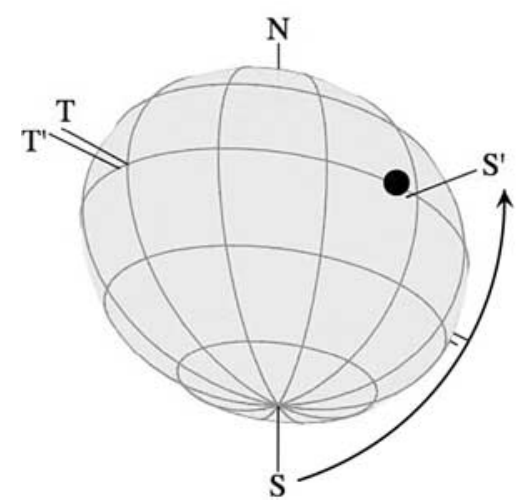

(B) Final rotation and tidal axes reference frame

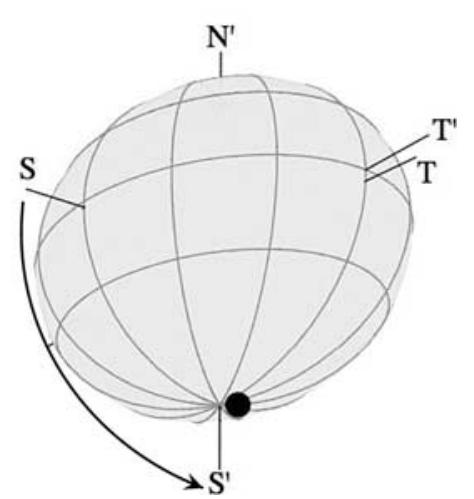

Figure 4. Reorientation of Enceladus driven by an internal load (filled circle) with $Q=-2$ in a reference frame aligned with the (a) initial (before reorientation) and (b) final (after reorientation) rotation and tidal axes. The initial north pole, south pole, and tidal axis are labeled with the letters $\mathrm{N}, \mathrm{S}$, and $\mathrm{T}$, respectively. The final north pole, south pole, and tidal axis are labeled with the letters $\mathrm{N}^{\prime}, \mathrm{S}^{\prime}$, and $\mathrm{T}^{\prime}$, respectively. The arrow indicates the direction of reorientation. Grid lines indicate latitude and longitude in $30^{\circ}$ increments. In the initial reference frame (Figure $4 \mathrm{a}$ ), the coordinates for the load (filled circle), the final south pole $\left(\mathrm{S}^{\prime}\right)$, and the final tidal axis $\left(\mathrm{T}^{\prime}\right)$ are $1^{\circ} \mathrm{S}, 80^{\circ} \mathrm{E}\left(\theta_{L}=91^{\circ}, \phi_{L}=80^{\circ}\right) ; 2^{\circ} \mathrm{S}, 86^{\circ} \mathrm{E}$; and $0^{\circ}$ $\mathrm{N}, 4^{\circ} \mathrm{W}$, respectively. In the final reference frame (Figure $4 \mathrm{~b}$ ), the coordinates for the load (filled circle), the initial south pole $(\mathrm{S})$, the initial north pole, and the initial tidal axis $(\mathrm{T})$ are $84^{\circ} \mathrm{S}, 10^{\circ} \mathrm{E}\left(\theta_{L}^{f}=174^{\circ}\right.$, $\left.\phi_{L}^{f}=10^{\circ}\right) ; 2^{\circ} \mathrm{S}, 90^{\circ} \mathrm{W} ; 2^{\circ} \mathrm{N}, 90^{\circ} \mathrm{E}\left(\theta_{R}=88^{\circ}, \phi_{R}=90^{\circ}\right)$; and $4^{\circ} \mathrm{S}, 0^{\circ} \mathrm{E}\left(\theta_{T}=94^{\circ}, \phi_{T}=0^{\circ}\right)$, respectively. The reorientation angle is $88^{\circ}$, and the angular distance between the load and the final south pole is $6^{\circ}$.

Even larger loads (Figures $3 \mathrm{a}$ and $3 \mathrm{~d}$ ) permit similar results for a broader range of initial load longitudes.

[31] An example of the kind of reorientation which may have occurred on Enceladus, if the load is sufficiently large, is given in Figure 4. A load originally located near the center of the leading/trailing hemisphere results in reorientation of $71^{\circ}$ and results in the load at a distance of $9^{\circ}$ from the rotation axis. On the basis of geological observations of equatorial features that resemble the polar "tiger stripes" tectonic features, it has been suggested [Helfenstein et al., 2006; P. Helfenstein et al., Patterns of tectonism and polar wander on Enceladus, submitted to Icarus, 2007; hereinafter referred to as Helfenstein et al., submitted manuscript, 2007] that Enceladus may have undergone more than one episode of reorientation. These older equatorial features are located at approximately $0^{\circ} \mathrm{N}, 80^{\circ}$ E. Figure 4 shows a potential reorientation scenario which may explain the current location of these features. Assume that the ancient equatorial features began at the initial south pole (perhaps due to another TPW event). Now let a new load with $Q=-2$ develop near the leading hemisphere $\left(\theta_{L}=91^{\circ}, \phi_{L}=80^{\circ}\right)$, as shown in Figure $4 \mathrm{a}$. Reorientation will bring the rotation axis to within $6^{\circ}$ of the new load, with the load ultimately positioned at $84^{\circ} \mathrm{S}, 10^{\circ} \mathrm{E}\left(\theta_{L}^{f}=174^{\circ}, \phi_{L}^{f}=10^{\circ}\right)$ in the final coordinate system aligned with the final rotation and tidal axes (Figure 4b). Given these final coordinates, equations (32)-(35) can be used to calculate the coordinates for the initial rotation pole in the final reference frame. These coordinates are $2^{\circ} \mathrm{N}, 90^{\circ} \mathrm{E}\left(\theta_{R}=88^{\circ}, \phi_{R}=90^{\circ}\right)$, which are similar to the present-day observed location of the hypothesized ancient polar terrain $\left(0^{\circ} \mathrm{N}, 80^{\circ} \mathrm{E}\right)$. Thus an initially polar region can be reoriented to an equatorial locality by the application of a sufficiently large load located near the center of the leading hemisphere.

[32] For the case of Enceladus, we may therefore draw the following conclusions. If the load is small $(Q \approx-1)$, significant reorientation is ruled out because of the small present-day angular separation between the load and the rotation axis (Figures $3 \mathrm{c}$ and $3 \mathrm{f}$ ). On the other hand, a larger load $(Q \approx-2)$ permits reorientations approaching $90^{\circ}$ (Figures $3 \mathrm{~b}$ and $3 \mathrm{~d}$ ). Larger reorientations are favored if the initial load longitude is close to $90^{\circ}$, that is, close to the center of the leading or trailing hemisphere. Finally, the TPW scenario shown in Figure 4 is also consistent with the present-day equatorial location of a hypothesized ancient polar terrain.

\section{Discussion}

[33] We have derived TPW solutions for tidally deformed planetary bodies by diagonalizing the component of inertia tensor perturbation which does not follow the rotation and tidal axes, which we referred to as the non-equilibrium inertia tensor (equation (27)). The rotation pole is stabilized by the remnant rotational and tidal bulges, and since the latter dominates the non-equilibrium inertia tensor, reorientation is more easily accomplished around the tidal axis. The TPW solutions with tidal deformation predict less permissive ranges of reorientation relative to the solutions which do not include this effect.

[34] We applied the new theory to consider the rotational stability of Enceladus in response to loading by an internal load. Small loads $(|Q| \approx 1)$ do not permit significant reorientation because of the small present-day angular separation between the load and the rotation axis. Larger 
loads $(|Q| \approx 2)$ permit reorientations approaching $90^{\circ}$. The latter is favored if the initial load longitude is close to $90^{\circ}$ or $270^{\circ}$, that is, close to the center of the leading or trailing hemisphere of the satellite. These regions experience more tidal heating in a thin shell than do the sub- and anti-Saturn points, though less than the polar regions [Ojakangas and Stevenson, 1989a, Figure 1]. Furthermore, the present-day equatorial location of a hypothesized ancient polar terrain [Helfenstein et al., 2006; Helfenstein et al., submitted manuscript, 2007] is consistent with a $\sim 90^{\circ}$ TPW event driven by a large load initially located near the center of the leading hemisphere.

[35] In this work we have assumed that the near-surface of the planetary body can maintain stresses over geological timescales, that is, there is a lithosphere resulting in remnant rotational and tidal bulges which oppose reorientation. Because most planetary bodies have cold, rigid surfaces, this assumption is likely appropriate; nonetheless, it is possible that lithospheric relaxation may be important. If the lithosphere behaves viscoelastically and the load is applied over time scales that are longer than the viscous relaxation time scale, the remnant rotational and tidal bulges will eventually adjust to any new rotation and tidal axes orientation. Hence the final rotation axis orientation is governed by the non-hydrostatic inertia tensor (which depends on the load alone). In this case, TPW driven by a negative load ultimately aligns the rotation axis with the load, while a positive load ultimately drives itself to the equator, irrespective of the initial load location.

[36] As a caveat, we note that reorientation without stabilization by remnant rotational and tidal bulges may occur, even in the presence of an elastic lithosphere, for satellites with a subsurface ocean. In this case, the interior may decouple from the lithospheric shell, enabling TPW of the (decoupled) interior driven by an internal load to proceed without stabilization by the remnant rotational and tidal bulges, and the rotation axis is ultimately aligned with the internal load. This may explain the close alignment between Enceladus' hot spot and the south pole; however, it cannot explain the geologically inferred ancient polar terrain [Helfenstein et al., 2006; Helfenstein et al., submitted manuscript, 2007] since the decoupled lithosphere remains fixed during reorientation of the interior. If Enceladus has a subsurface ocean, the observed tectonic pattern may be explained by reorientation of the floating shell due to variations in its thickness [Ojakangas and Stevenson, 1989b].

[37] Whether or not Enceladus has a subsurface ocean, given its observed shape [Thomas et al., 2007], a large reorientation $\left(\sim 90^{\circ}\right)$ of the lithosphere results in strains $\sim 10^{-2}$, large enough for ice failure and plastic flow to occur [Rist, 1997]. Hence reorientation is likely to produce global tectonic patterns on the lithosphere [Vening Meinesz, 1947; Melosh, 1980]. Stabilization by the remnant rotational and tidal bulges is expected to be smaller, but nonzero, for a broken lithosphere with a plastic rheology. Tectonic features in current or future Cassini images may provide constraints for the possible reorientation scenarios. Additionally, the gravitational signal of the load and the remnant rotational and tidal bulges may be detectable with Cassini.
[38] Acknowledgments. The authors thank H. J. Melosh and B. G. Bills for helpful comments. We acknowledge support from the Carnegie Institution of Washington and the NASA Outer Planets Research.

\section{References}

Argus, D. F., and R. S. Gross (2004), An estimate of motion between the spin axis and the hotspots over the past century, Geophys. Res. Lett., 31, L06614, doi:10.1029/2004GL019657.

Besse, J., and V. Courtillot (2002), Apparent and true polar wander and the geometry of the geomagnetic field over the last $200 \mathrm{Myr}, J$. Geophys. Res., 107(B11), 2300, doi:10.1029/2000JB000050.

Bills, B. G., and T. S. James (1999), Moments of inertia and rotational stability of Mars: Lithospheric support of subhydrostatic rotational flattening, J. Geophys. Res., 104(E4), 9081-9096.

Darwin, G. H. (1877), On the influence of geological changes on the Earth's axis of rotation, Philos. Trans. R. Soc. London, 167, 271-312.

Evans, J. (1866), On a possible geological cause of changes in the position of the axis of the earth's crust, Proc. R. Soc. London, 15, 46-54.

Gold, T. (1955), Instability of the Earth's axis of rotation, Nature, 175 $526-529$.

Goldreich, P., and A. Toomre (1969), Some remarks on polar wandering, J. Geophys. Res., 74, 2555-2567.

Greff-Lefftz, M. (2004), Upwelling plumes, superswells and true polar wander, Geophys. J. Int., 159, 1125-1137, doi:10.1111/j.1365246X.2004.02440.x

Helfenstein, P., P. C. Thomas, J. Veverka, C. Porco, B. Giese, R. Wagner, T. Roatsch, T. Denk, G. Neukum, and E. Turtle (2006), Surface geology and tectonism on Enceladus, Eos Trans. $A G U, 87(52)$, Fall Meet. Suppl., Abstract P22B-02.

Kaula, W. M. (1968), An Introduction to Planetary Physics-The Terrestrial Planets, Space Science Text Series, John Wiley, New York.

Kirschvink, J. L., R. L. Ripperdan, and D. A. Evans (1997), Evidence for a large-scale reorganization of early Cambrian continental masses by inertial interchange true polar wander, Science, 277(5325), 541-545, doi:10.1126/science.277.5325.541.

Love, A. E. H. (1911), Some Problems of Geodynamics, Cambridge University Press, Cambridge, UK.

Matsuyama, I., J. X. Mitrovica, M. Manga, J. T. Perron, and M. A. Richards (2006), Rotational stability of dynamic planets with elastic lithospheres, J. Geophys. Res., 111, E02003, doi:10.1029/2005JE002447.

Melosh, H. J. (1980), Tectonic patterns on a reoriented planet-Mars, Icarus, 44, 745-751, doi:10.1016/0019-1035(80)90141-4.

Murray, C., and S. Dermott (1999), Solar System Dynamics, Cambridge Univ. Press, New York.

Nimmo, F., and R. T. Pappalardo (2006), Diapir-induced reorientation of Saturn's moon Enceladus, Nature, 441, 614-616, doi:10.1038 nature04821.

Ojakangas, G. W., and D. J. Stevenson (1989a), Thermal state of an ice shell on Europa, Icarus, 81, 220-241, doi:10.1016/00191035(89)90052-3.

Ojakangas, G. W., and D. J. Stevenson (1989b), Polar wander of an ice shell on Europa, Icarus, 81, 242-270, doi:10.1016/0019. 1035(89)90053-5.

Porco, C. C., et al. (2006), Cassini observes the active south pole of Enceladus, Science, 311, 1393-1401, doi:10.1126/science.1123013.

Ricard, Y., G. Spada, and R. Sabadini (1993), Polar wandering of a dynamic earth, Geophys. J. Int., 113, 284-298.

Richards, M. A., Y. Ricard, C. Lithgow-Bertelloni, G. Spada, and R. Sabadini (1997), An explanation for Earth's long-term rotational stability, Science, 275(5298), 372-375, doi:10.1126/science.275.5298.372.

Richards, M. A., H.-P. Bunge, Y. Ricard, and J. R. Baumgardner (1999), Polar wandering in mantle convection models, Geophys. Res. Lett., 26(12), $1777-1780$.

Rist, M. (1997), High-stress ice fracture and friction, J. Phys. Chem. B, 101(32), 6263-6266, doi:10.1021/jp963175x.

Spada, G., Y. Ricard, and R. Sabadini (1992), Excitation of true polar wander by subduction, Nature, 360, 452-454

Spada, G., R. Sabadini, and E. Boschi (1996), Long-term rotation and mantle dynamics of the Earth, Mars, and Venus, J. Geophys. Res., 101(E1), 2253-2266

Spencer, J. R., J. C. Pearl, M. Segura, F. M. Flasar, A. Mamoutkine, P. Romani, B. J. Buratti, A. R. Hendrix, L. J. Spilker, and R. M. C. Lopes (2006), Cassini encounters Enceladus: Background and the discovery of a south polar hot spot, Science, 311, 1401-1405, doi:10.1126/ science.1121661.

Sprenke, K. F., L. L. Baker, and A. F. Williams (2005), Polar wander on Mars: Evidence in the geoid, Icarus, 174, 486-489, doi:10.1016/ j.icarus.2004.11.009. 
Steinberger, B., and R. J. O'Connell (1997), Changes of the Earth's rotation axis owing to advection of mantle density heterogeneities, Nature, 387, $169-173$.

Thomas, P. C., et al. (2007), Shapes of the Saturnian icy satellites and their significance, Icarus, 190, 573-584, doi:10.1016/j.icarus.2007.03.012.

Vening Meinesz, F. A. (1947), Shear patterns of the Earth's crust, Eos Trans. $A G U, 28,939-946$.

Willemann, R. J. (1984), Reorientation of planets with elastic lithospheres, Icarus, 60, 701-709, doi:10.1016/0019-1035(84)90174-X.
Zuber, M. T., and D. E. Smith (1997), Mars without Tharsis, J. Geophys. Res., 102(E12), 28,673-28,686.

I. Matsuyama, Department of Terrestrial Magnetism, Carnegie Institution of Washington, 5241 Broad Branch Road, NW, Washington, DC 20015 USA. (matsuyama@dtm.ciw.edu)

F. Nimmo, Department of Earth and Planetary Sciences, University of California, Santa Cruz, 1156 High Street, Santa Cruz, CA 95064, USA. 